Ann. Sci. forest., 1980, 37 (1), 19-36.

\title{
Variabilité de l'angle du fil du bois chez quelques feuillus : Hêtre, Chêne et Eucalyptus dalrympleana
}

\author{
Y. BIROT *, J. DUFOUR *, P. FERRANDES **, E. TEISSIER du CROS *, \\ P. AZOEUF *** ef R. HOSLIN *** \\ * Station d'Amélioration des Arbres Forestiers \\ Centre de Recherches forestières d'Orléans, I.N.R.A., \\ Ardon, 45160 Olivet \\ ** Station Expérimentale des Maures, I.N.R.A. \\ Le Ruscas, R. N. 98 \\ 83230 Bormes les Mimosas \\ *** Commissariat d̀ l'énergie atomique \\ Section d'Application de la Radio-Activité \\ B. P. n 2, 91190 Gif-sur-Yvette
}

\begin{abstract}
Résumé
La fibre torse est considérée généralement comme un défaut grave pour la plupart des utilisations du bois. Des mesures de l'angle de déviation des fibres ont été réalisées à différentes hauteurs dans l'arbre pour trois essences feuillues : Eucalyptus dalrympleana, Fagus silvatica ef Quercus petraea. Les mesures ont été effectuées d'une part grâce à l'injection de Potassium ${ }^{42} \mathrm{~K}$ sous écorce, d'autre part, sur écorce, d'après photographies : 1) A 1,30 m les valeurs extrêmes observées sont fortes chez l'Eucalyptus ( 27 grades) plus faibles chez le Hêtre et le Chêne (respectivement 13 et 10 grades). 2) L'angle de déviation du fil du bois est variable avec la hauteur en particulier pour l'Eucalyptus, mais les valeurs observées aux différents niveaux dans l'arbre sont significativement corrélées : une mesure à $1,30 \mathrm{~m}$ permet donc de juger l'ensemble du fût. 3) Pour les trois espèces étudiées l'observation de l'écorce à l'œil nu est suffisante pour détecter les arbres présentant une forte déviation, résultat qui intéresse particulièrement le forestier de terrain. 4) Pour l'Eucalyptus, on a pu montrer que l'effet famille étaił peu élevé sur ce caractère. Deux questions mériteraient d'être approfondies à l'issue de cette étude : a) évolution de l'angle de déviation, en fonction de l'âge à un niveau donné dans l'arbre ; b) transmissibilité héréditaire du caractère.
\end{abstract}

\section{1. - Introduction}

Une déformation importante de l'angle des éléments constitutifs du bois à un niveau donné, par rapport aux segments de génératrices $\left(^{1}\right)$ de ces niveaux est appelée fibre torse dans le langage forestier classique. Elle est défavorable à l'utilisation de ce matériau sous forme de sciages, ou de feuillets de placage obtenus par tranchage ou déroulage. Par la suite, cette notion sera désignée par l'«angle du fil du bois ».

(1) Les portions de fût d'environ $30 \mathrm{~cm}$ de haut au niveau desquelles sont réalisées les observations sont assimiées à des troncs de cône. Le terme de « génératrice » a donc une définition géométrique. 
Les études récentes menées en France sur ce thème grâce à l'utilisation de traceurs radioisotopes ont concerné essentiellement des conifères d'importance économique majeure : Douglas (Pseudotsuga menziesii, Mirb. Franco), Pin maritime (Pinus pinaster Ait.) et Pin laricio (Pinus nigra ssp. laricio Arnold). Elles avaient pour objectif de préciser un certain nombre de points : variabilité phénotypique (à $1,30 \mathrm{~m})$, variabilité dans l'espace (le long d'un même cerne à différents niveaux), variabilité dans le temps à un niveau donné (de la moelle vers l'écorce), variabilité génétique.

Pour le Pin laricio, l'hérédité de l'angle du fil du bois serait surtout de type additif, alors que pour le Pin maritime une certaine part serait dûe à des effets génétiques non additifs (Arbez et al., 1978). Pour le Douglas et le Pin laricio l'angle du fil du bois des cernes externes augmente avec la hauteur (Birot ef al., 1979), cependant les variations intra arbres sont anarchiques : il n'y a pas de corrélation entre niveaux. Par ailleurs, il n'y a, pour ces trois espèces, aucune relation entre l'aspect extérieur du rhyditome et l'angle du fil du bois.

L'objectif des études dont les résultats sont présentés ici, esł d'étendre ce type d'observations à certains feuillus réputés pour ce défaut de fibre torse : le Chêne (Quercus petraea (Mattushka) Lieblein), le Hêtre (Fagus silvatica L.) et Eucalyptus dalrympleana Maiden.

Pour les deux premières espèces, dont la régénération est en majorité assurée par voie naturelle, il est important de connaître le déterminisme génétique de l'angle du fil du bois de façon à orienter le choix des semenciers au moment des éclaircies. II en est de même si l'on envisage la création de peuplements porte-graines, ou tout simplement la sélection individuelle dans le cadre de programmes d'amélioration. Pour le Chêne et le Hêtre, les limites tolérables pour les utilisateurs du bois seraient les suivantes d'après Krahl-Urban (1953) :

$$
\begin{aligned}
& \text { Chêne : sciage : } 10^{\circ} \text { - tranchage : } 15^{\circ} \\
& \text { Hêtre : sciage : } 10^{\circ} \text { - déroulage : } 6^{\circ} \text { environ. }
\end{aligned}
$$

Pour Venef (1970), un angle supérieur à 2 grades serait déjà défavorable chez le Chêne. Il précise par ailleurs (communication personnelle en 1977) que « lorsque la fibre torse du Hêtre ne dépasse pas dix grades, elle n'a pas d'incidence sur la fabrication des contreplaqués, en particulier des contreplaqués emballages. Lorsque la fibre torse dépasse dix grades, il n'y a pas non plus d'incidence sur l'utilisation mais cela entraîne une réfaction sur les prix qui peut atteindre 25 p. 100 ».

Eucalyptus dalrympleana s'est révélé comme une espèce très prometteuse (résistance au froid, rapidité de croissance) en zone méditerranéenne française (Ferrandes, 1970). Mais, en plus de phénomènes de retrait ef de collapse, classiques chez les Eucalyptus, cette espèce, tout au moins dans le matériel introduit en France, présente une fibre torse marquée (Polge, communication personnelle). Un programme de sélection individuelle débouchant sur la création d'un verger à graines de clones a été engagé. Les critères de sélection retenus sont les suivants : résistance au froid, croissance rapide, faible angle du fil du bois.

Pour les trois espèces étudiées, la conformation de l'écorce donne chez certains individus l'impression de refléter la déviation de l'angle du fil du bois sous-jacent. Les figures 1 et 2 en donnent des exemples chez le Chêne et le Hêtre. Chez Eucalyptus dalrympleana dont l'écorce est à grain très fin, la même apparence existe mais est plus difficile à photographier. 
FIG. 1. - Un Hêtre à fibre torse. A Beech tree with spiral grain
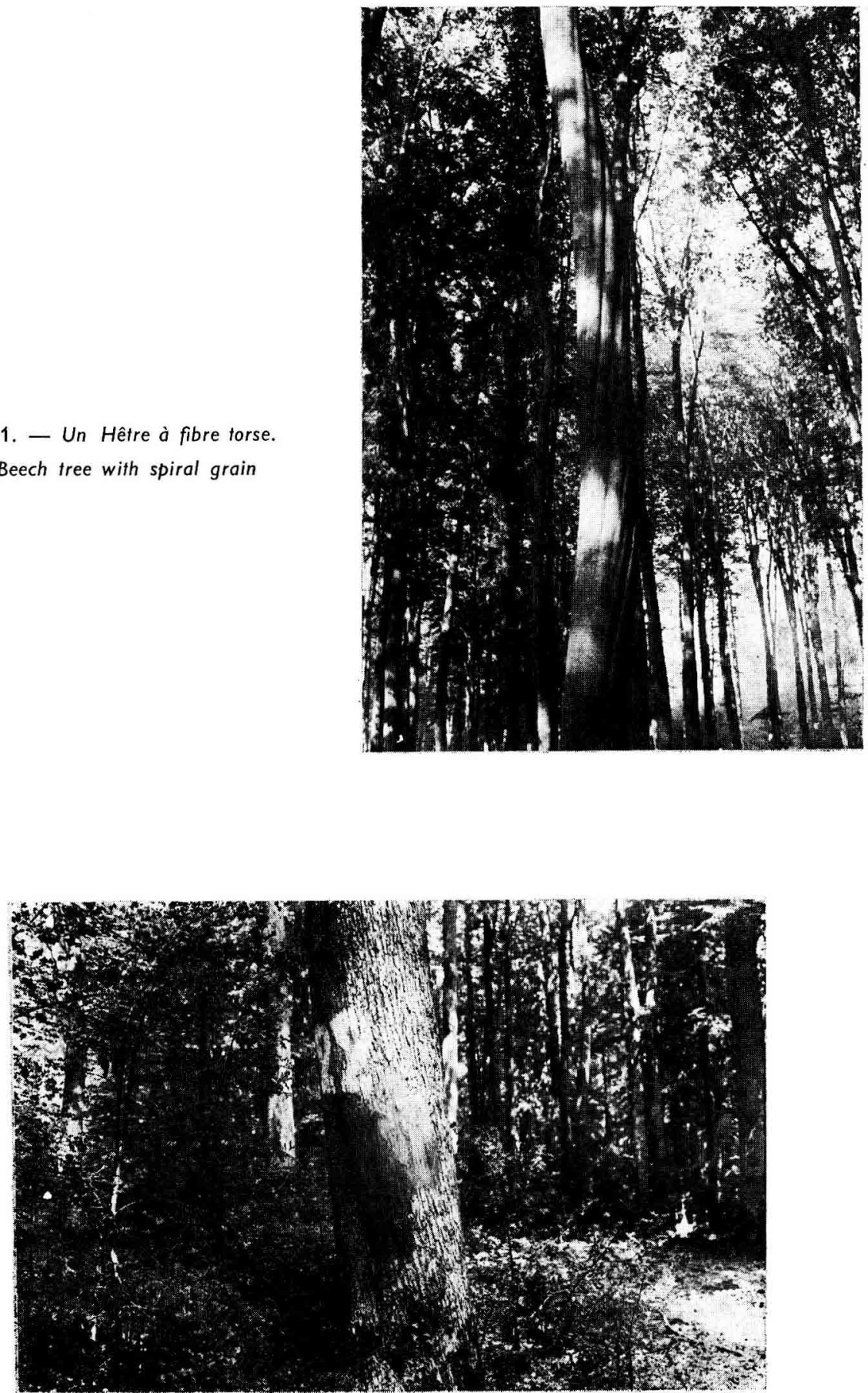

FIG. 2. - Un Chêne à fibre torse.

An Oak tree with spiral grain. 
Les principales questions posées dans le cadre de cette étude sont donc les suivantes :

- variabilité phénotypique de l'angle du fil du bois à un niveau donné ;

- relation entre l'angle du fil du bois sous écorce, et l'aspect apparent sur écorce;

- variabilité avec la hauteur de l'angle du fil du bois des cernes extérieurs ${ }^{(1)}$ : contraste d'angle du fil du bois sur un fût ;

- hérédité de l'angle du fil du bois.

\section{2. - Matériels et méthodes}

\section{1. - Matériel végétal}

Pour le Hêtre, l'étude a été réalisée dans une futaie régulière pure d'environ 100 ans en forêt domaniale de Lyons (Haute-Normandie). Quarante arbres représentant la variabilité du peuplement pour des caractéristiques générales de forme, ont été échantillonnés. La déviation de la fibre a été appréciée pour 20 d'entre eux à $1 \mathrm{~m}$, $5 \mathrm{~m}$ ef $9 \mathrm{~m}$ et pour les vingt autres à $1 \mathrm{~m}$ seulement.

Pour le Chêne rouvre, les observations ont eu lieu en forêt domaniale de Bercé (Sarthe). La parcelle étudiée est une futaie régulière d'environ 130 ans où ce Chêne domine. L'échantillon et les niveaux d'injection sont les mêmes que pour le Hêtre.

Les quarante arbres choisis pour chacune de ces deux espèces font partie d'un échantillon plus important (environ 70) inclus dans une étude de structure génétique. Cette étude est destinée à mettre en évidence les parts relatives de l'hérédité et du milieu dans le déterminisme de différents caractères d'importance économique : phénologie, forme, qualité du bois, résistance aux maladies. A l'avenir lorsque les descendances individuelles de ces arbres déjà en cours d'élevage et leurs copies végétatives seront suffisamment développées, l'angle de déviation de leur fibre sera aussi mesuré. II nous apportera des estimations des héritabilités génotypiques et génétiques de ces caractères.

Pour l'Eucalyptus, les observations ont porté sur un test de provenances $x$ descendances (maternelles) situé en forêt communale de Pierrefeu (Var), en dispositif à parcelles mono-arbre.

Cette expérience installée en 1966 compare sept provenances représentées chacune par $n$ descendances maternelles $(4<n<10)$. Sur la base des critères de sélection mentionnés précédemment incluant le faible angle du fil du bois, on a sélectionné trente sept arbres appartenant à sept provenances. L'angle du fil du bois a été mesuré sur ces individus.

Par ailleurs, et uniquement dans une provenance (Tantangara, Australie, altitude $1200 \mathrm{~m}$, latitude $35^{\circ} 45^{\prime}$, longitude $148^{\circ} 30 \mathrm{E}$ ), on a mesuré la fibre torse à $1,30 \mathrm{~m}$ sur 13 individus pris au hasard dans chacune des dix familles (au total cent seize arbres ont été mesurés) et si possible dans les mêmes blocs ou dans des blocs adjacents. Sur environ neuf de ces individus par famille (au total sur quatre-vingt-huit arbres),

(1) L'étude de l'évolution de l'angle du fil du bois (du cœur vers l'écorce) ne pouvait êire réalisée avec la méthode 
on a mesuré la fibre torse à un deuxième niveau : $4 \mathrm{~m}$. La hauteur moyenne des arbres au moment des mesures était d'environ $8 \mathrm{~m}$.

\section{2. - Méthode.}

La méthode d'appréciation de l'angle de déviation du fil du bois par rapport à l'axe du fût, utilisée dans cette étude, est non destructive pour les arbres. Elle met en œuvre l'autoradiographie de la trace suivie par un radioisotope, le ${ }^{42}$ Potassium, injecté sous forme de chlorure, dans les couches périphériques du bois en période d'active ascension de sève. Elle a été décrite en détail par Keller et al. en 1974, puis rappelée par Arbez et al. en 1978 et Birot ef al. en 1979.

La seule modification que nous ayons apportée réside dans le tracé des génératrices qui a été déterminé optiquement au moyen d'un tachéomètre. Cette méthode a été employée pour le Hêtre et le Chêne rouvre (voir annexe I). Pour les Eucalyptus la méthode est celle du compas «azimuté » présentée par Biroł ef al. (op. cit.).

Pour le Chêne et le Hêtre, l'appréciation sur écorce de la déviation du fil du bois a été réalisée au moyen de photos prises au niveau d'injection du ${ }^{42} \mathrm{~K}$. Les mesures d'angles ont été faites sur des projections de ces photos. La direction de la déviation de l'écorce est un faisceau de droites parallèles fictives qui, dans le cas du Chêne, intègrent la direction générale des fissures, et dans le cas du Hêtre, matérialisent la direction des défauts longitudinaux : cannelures, dépressions, fentes, La direction de référence est la génératrice du niveau de mesure lorsqu'elle est visible sur la photo, ou à défaut, la moyenne des angles d'inclinaison des deux plans tangentiels à l'arbre et passant par l'objectif de l'appareil photographique. Nous n'avons pas tenu compte de l'erreur de parallaxe due à la prise de photos vers les niveaux 5 et $9 \mathrm{~m}$, pour nous placer dans le cas normal d'un observateur debout.

Chez l'Eucalyptus, la déviation de l'écorce n'a pas éłé estimée de façon quantitative du fait de la finesse des fissures de l'écorce.

\section{3. - Résultats}

3.1. - Le Hêtre

Les observations ont été réalisées du 21 au 23 juin 1977.

\subsection{Variabilité du caractère.}

Mesures radiographiques.

La figure 3 donne les effectifs par niveau et par classe d'angle de déviation. Les limites des classes ont été fixées en tenant comple des critères des utilisateurs (cf. introduction) : à droite comme à gauche, 0 à 5 grades, 6 à 10 grades, plus de 10 grades (réfaction sur les prix). La zone sans pointillé sur celte figure correspond aux vingt arbres analysés à trois niveaux. Parmi les quarante arbres mesurés à $1 \mathrm{~m}$, trente trois ont une déviation inférieure ou égale à 5 grades $(82,5$ p. 100) ; un seul a une déviation qui dépasse 10 grades. 
Toujours à $1 \mathrm{~m}$ de hauteur, si on ne tient plus comple des classes mais des valeurs réelles, on constate que vingt quatre arbres dévient à droite, 6 à gauche et 10 ne montrent aucune déviation. Il semblerait donc que dans le peuplement analysé on rencontre une majorité de fibre torse déviant à droite.

HETRE
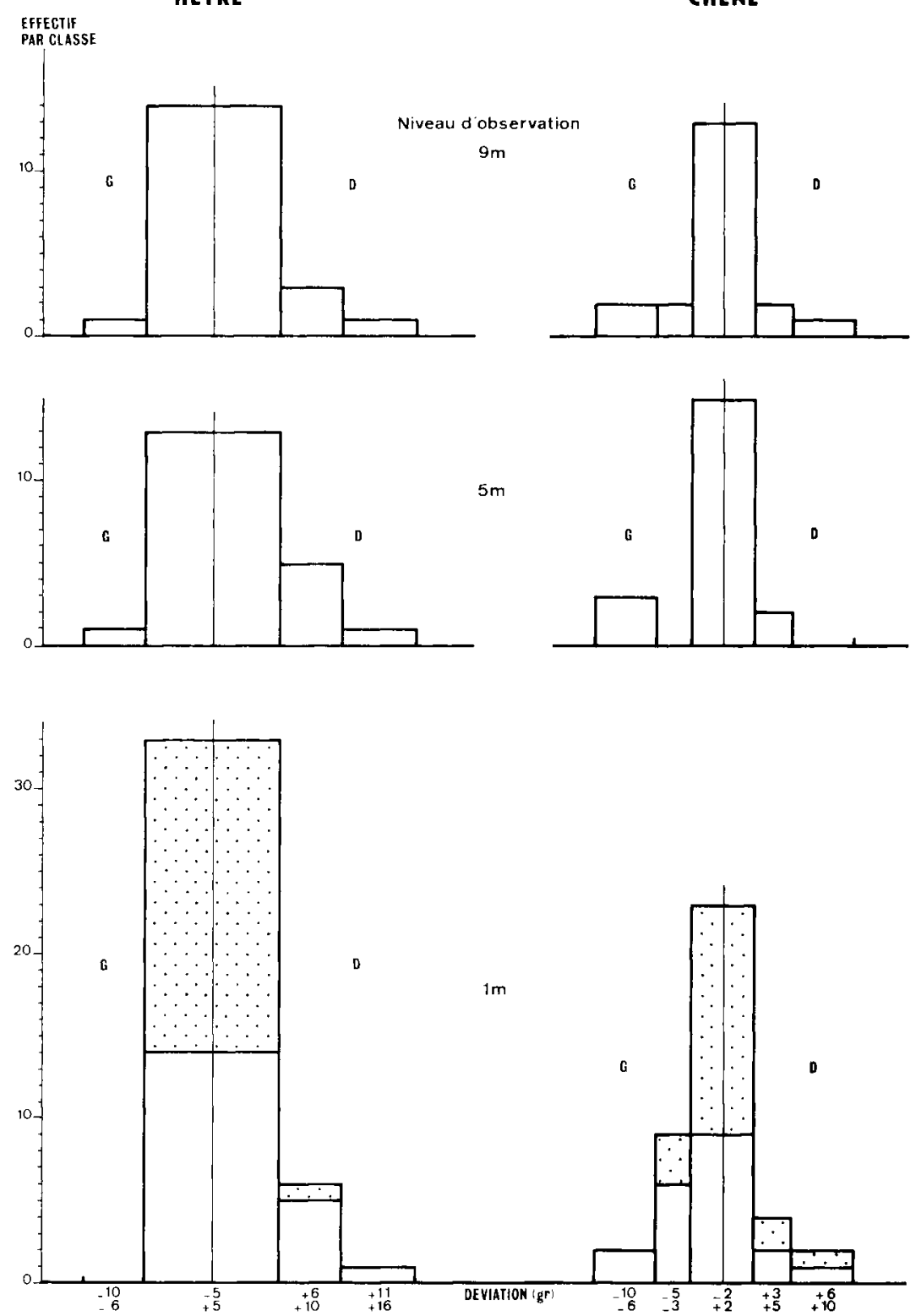

ChENE
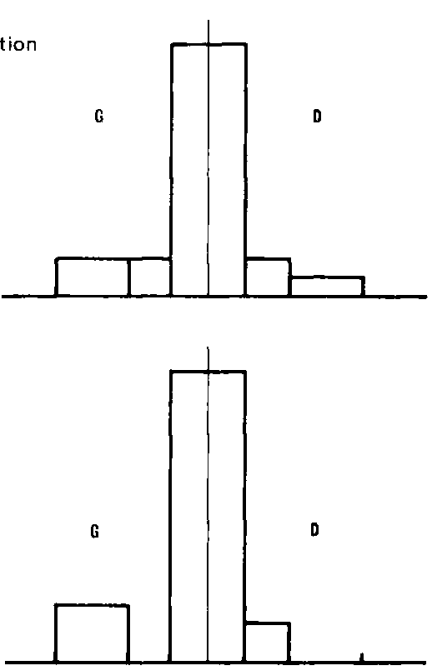

FIG. 3. - Hêtre et Chène : nombre d'individus par classe d'angle de déviation ; la partie en pointillés représente les arbres n'ayant été mesurés qu'à $1 \mathrm{~m}$ de haut : $G$ : gauche ; $D$ : droite.

Beek and Oak : number of trees per class of deviation angle; the part with dots represents trees measured only at 1 meter level : $G$ : left ; $D$ : right. 
La variation de l'angle du fil du bois avec la hauteur est représentée par la figure 4. Que ce soit pour la moyenne de tous les arbres, pour celle des arbres qui dévient à droite ou pour celle des arbres qui dévient à gauche, la déviation se déporte moins à droite ou, ce qui revient au même, plus à gauche lorsqu'on s'élève dans l'arbre.

En moyenne, cette modification de la déviation en fonction de la hauteur n'est pas élevée puisqu'elle n'atteint que 0,9 grade entre 1 et $9 \mathrm{~m}$, pour les arbres déviant à droite, 1,6 grades pour l'ensemble des vingt arbres et 2,7 grades pour les arbres déviant à gauche.

Au niveau individuel par contre, la variation de la déviation en fonction de la hauteur est légèrement plus élevée puisqu'elle peut atłeindre 5 d̀ 6 grades entre deux mesures voisines.

Il nous importait à ce stade de connaître la liaison statistique entre les différents niveaux de mesure pour savoir si une observation réalisée à un niveau quelconque dans l'arbre permet de prédire la déviation de la fibre de l'ensemble de l'arbre. Les corrélations entre les mesures des trois niveaux ont été calculées pour les vingt arbres (tabl. 1).

\section{TABLEAU 1}

Hêtre ef Chêne : coefficient de corrélation entre les trois niveaux de mesures

Beech and Oak : correlation coefficient befween the three measurement levels

\begin{tabular}{ccccc} 
Niveau $(\mathrm{m})($ Level $(\mathrm{m}))$ & \multicolumn{1}{c}{ Hêtre (Beech) } & \multicolumn{2}{c}{ Chêne (Oak) } \\
\hline 5 & 1 & 5 & 1 & 5 \\
9 & $0,84 * *$ & & $0,85 * * *$ & $0,89 * * *$ \\
\hline
\end{tabular}

Elles sont toutes très élevées. Une mesure réalisée à un niveau quelconque peut donc être considérée comme bonne prédictrice de la déviation de la fibre aux autres niveaux ; mais, compte tenu de la variation de la déviation avec la hauteur, on cherchera à faire une observation qui sera la limite supérieure de l'angle possible de déviation (fig. 4). Lorsqu'un arbre a une fibre torse à droite, une mesure en bas du fûł donnera en général la valeur la plus élevée, par contre chez un arbre à fibre torse à gauche, il faudra chercher à faire cette observation vers le haut du fût.

\section{Mesures sur photographies de l'écorce.}

Les photographies des troncs n'ont été réalisées que lorsqu'une déviation était visible à l'œil : cannelures (fig. 1), dépression sous une branche, fissures. Vingtneuf pholographies ont pu être prises. Les angles mesurés varient entre 5,6 grades à gauche et 18,3 grades à droite. Il faut remarquer que dans le peuplement étudié, le cas typique de la figure 1 est assez rare, il est impressionnant ; pourtant, quelle que soit la méthode employée les angles mesurés ne sont que de 7 à 9 grades; cet arbre serait donc, d'après les technologues, utilisable en déroulage. Mais il faut reconnaître qu'un tel aspect a tout lieu d'exercer un rôle psychologique défavorable sur l'acheteur. 


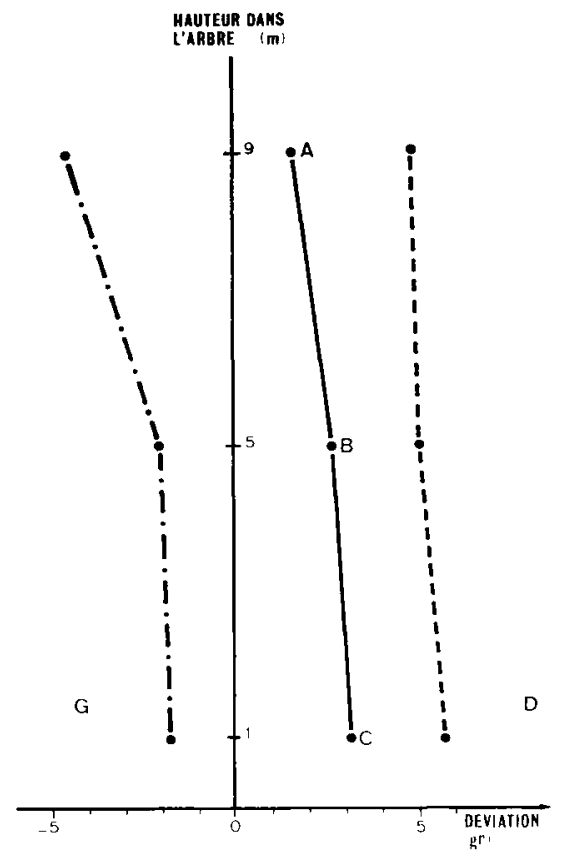

FIG. 4. - Hêtre : angle de déviation de la fibre en fonction de la hauteur de mesure sur le fût. $G:$ gauche ; $D$ : droite. La valeur moyenne à $9 \mathrm{~m}(\mathrm{~A})$ diffère statistiquement des deux autres $(\mathrm{B}$ ef $\mathrm{C})$, mais $\mathrm{B}$ et $C$ ne diffèrent pas entre elles. _- - - - - les 6 arbres ne déviant qu'à gauche ; - $-\ldots$ les 14 arbres ne déviant qu'à droite; ___ valeur moyenne pour les 20 arbres.

Beech : fiber deviation angle according to level of measurement on the bole. G : left ; D : right. The mean value at $9 \mathrm{~m}$ level $(A)$ is statistically different from the two others ( $B$ and $C$ ). But $B$ and $C$ do not differ from oneanother. - - - - - - 6 trees with leftward deviation ; - - -14 trees with rightward deviation ; mean value of 20 trees.

3.12. Validité de l'observation de la déviation de l'écorce pour apprécier celle du bois.

La figure 5 montre la liaison existant entre les deux méthodes de mesures pour les vingt neuf couples photo-radio tous niveaux confondus. Le coefficient de corrélation entre les deux distributions est de 0,81 ; il est significatif au seuil de 1 p. 1000 . Les forestiers pourront donc, au cours des martelages, éliminer avec une bonne efficacité les individus défectueux.

L'appréciation strictement visuelle de la déviation du bois par celle de l'écorce ne sera pourtant pas infaillible. En effet, dans noire échantillon, l'arbre pour lequel la méthode du ${ }^{42} \mathrm{~K}$ a permis de déceler l'angle de fibre torse le plus grand : 14 grades à $1 \mathrm{~m}$ et 13 grades à 5 et $9 \mathrm{~m}$, n'a montré sur écorce qu'une très vague déformation beaucoup plus haut que le niveau de mesure et détectable qu'avec un éclairage rasant du soleil. Si l'on peut admettre que ce phénomène est suffisamment rare pour être négligé dans une gestion courante d'une hêtraie, il devra être pris en considération dans les peuplements classés pour la production de faînes. Là, en effet, un petit nombre d'arbres produira une grande quantité de semences, chaque individu prenant une part importante de cette production. Cette dernière remarque suppose, bien entendu, que le défaut fibre torse est sous forte influence génétique, ce que nous sommes dans l'impossibilité d'affirmer encore chez le hêtre. 


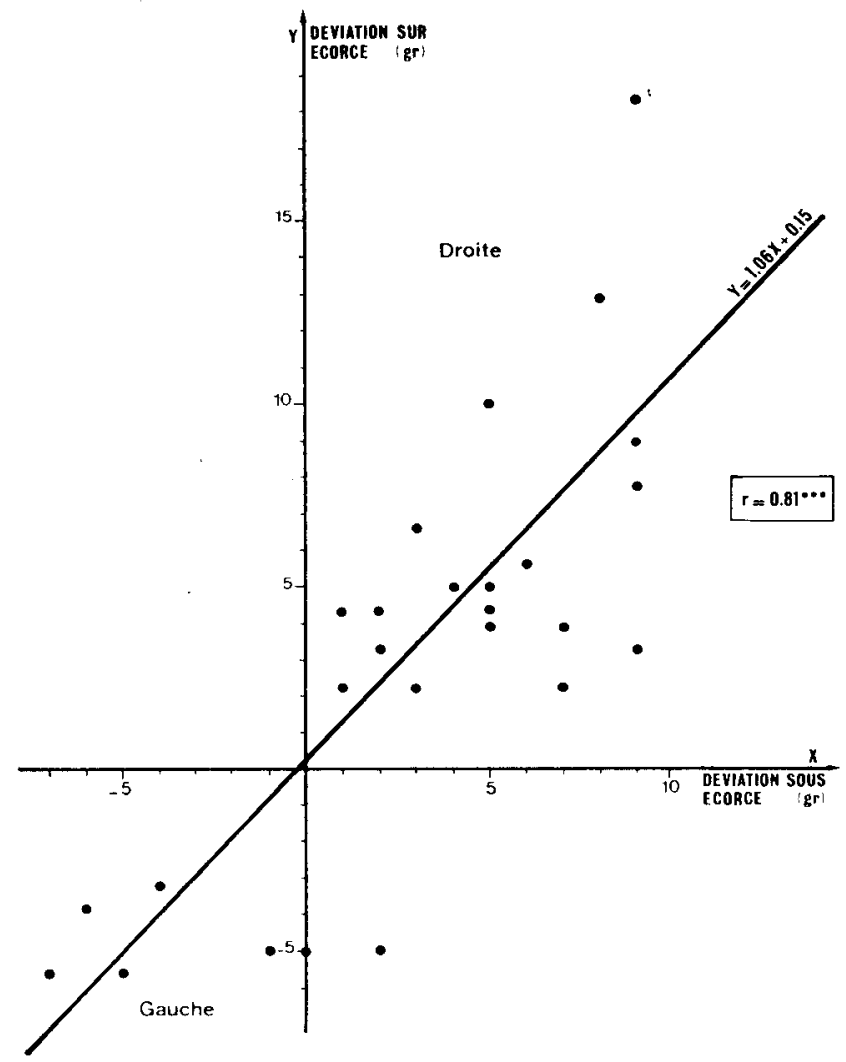

FIG. 5. - Hêtre : coefficient de corrélation ef droite de régression entre valeurs d'angles sur écorce et sous écorce, tous niveaux de mesures confondus.

Beech : correlotion coefficient and regression line between outside and inside bork fiber deviation values, including all measurement levels.

\section{2. - Le Chêne}

Les injections de potassium ${ }^{42} \mathrm{~K}$, la pose et l'enlèvement des films photo-sensibles, ontéleulieu entre le 29 mai et le 2 juin 1978.

\subsection{Variabilité du caractère.}

Sur l'échantillon observé en forêt de Bercé, le pourcentage d'arbres strictement de droit fil est relativement faible quel que soif le niveau observé $(12,5$ p. 100 à $1 \mathrm{~m}$; 20 p. 100 à $3 \mathrm{~m} ; 25$ p. 100 à $9 \mathrm{~m}$ ). Cependant, le phénomène n'afteint pas une très grande amplitude puisque l'angle de déviation des fibres ne dépasse jamais 10 grades. D'autre part, les angles mesurés sont le plus souvent inférieurs à 2 grades, les valeurs supérieures à 5 grades étant assez rares (fig. 3). Aucune tendance nette n'apparaît en ce qui concerne le sens de la déviation.

L'angle des fibres du bois est variable pour un même arbre en fonction de la hauteur. Dans deux cas sur 20 on a même observé un changement dans le sens de la déviation. Pour ces deux arbres, les mesures à $1 \mathrm{~m}, 5 \mathrm{~m}$ et $9 \mathrm{~m}$ sont respectivement : 
- 3 grades à droite, 1 grade à gauche, 4 grades à droite ;

- 3 grades à gauche, pas de déviation, 1 grade à droite.

La variation (ou contraste) de l'angle moyen de déviation est de 0,95 grade entre $1 \mathrm{~m}$ et $5 \mathrm{~m}$, elle est pratiquement nulle entre 5 et $9 \mathrm{~m}$ (fig. 6). Un test $\dagger$ (méthode des couples), montre que seul l'écart entre les moyennes à 1 et $5 \mathrm{~m}$ est significatif au seuil de probabilité $0,05(t=2,77$ pour $19 \mathrm{dl})$. Le coníraste d'angle entre les mesures individuelles reste inférieur à 4 grades, ce qui est assez faible.

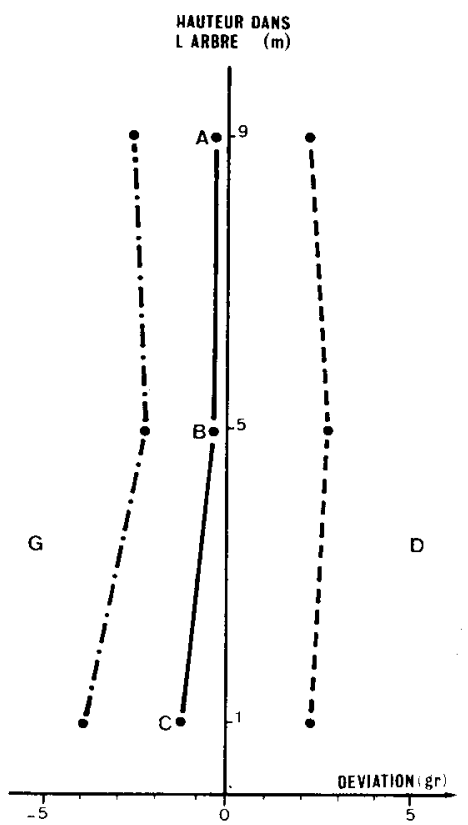

FIG. 6. - Chêne : angle de déviation de la fibre en fonction de la hauteur sur le fot. G. : gauche ; D : droite.

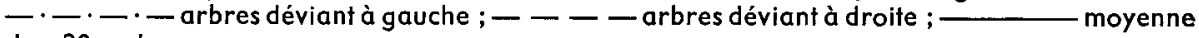
des 20 arbres.

Oak : fiber deviation angle according to level of measurement on the bole. $\mathrm{G}:$ left ; $\mathrm{D}$ : right. $-.-\cdots-\cdot-$ trees with leftward deviation ; - -1 trees with rightward deviation; - mean values of 20 trees.

Par ailleurs, les mesures de la fibre torse aux différentes hauteurs sont fortement corrélées (tabl. 1). Une observation à un niveau quelconque serait donc un bon indicateur de la valeur moyenne de la «torsion » sur l'ensemble du tronc. Cependant, les coefficients de régression des mesures à $1 \mathrm{~m}$ par rapport à celles à 5 et $9 \mathrm{~m}$ étant inférieurs à $1(0,725$ et 0,834$)$, la valeur observée à $1 \mathrm{~m}$ appliquée à l'ensemble de l'arbre serait en moyenne légèrement surestimée, ce qui réduit le risque d'erreur dans le cas d'une sélection phénotypique.

3.22. Validité des mesures sur écorce.

Les mesures d'angles sur écorce onł été faites sur l'ensemble de l'échantillon d'après photographies (cf. $§ 2.2$ ). Deux clichés peu nets ont été exclus de l'étude. Le coefficient de corrélation entre les estimations de l'angle de déviation sur écorce et 
sous écorce est de 0,865 (significatif au seuil 0,001 pour $76 \mathrm{dl}$ ). Le coefficient de .régression de la valeur sous écorce par rapport à la déviation de l'écorce est inférieur à $1(0,677)$; ceci indique qu'en moyenne dans notre échantillon nous avons sousestimé l'importance de la fibre torse en la mesurant sur écorce (fig. 7). Les mesures"sur écorce permettent donc de détecter facilement les arbres présentant une déviation importante du fil du bois. Une première notation rapide, analogue à celle que pourrait faire un forestier au cours d'un martelage, avait d'ailleurs permis de détecter tous les cas où la méthode par injection de ${ }^{42} \mathrm{~K}$ a révélé une valeur supérieure à 5 grades.

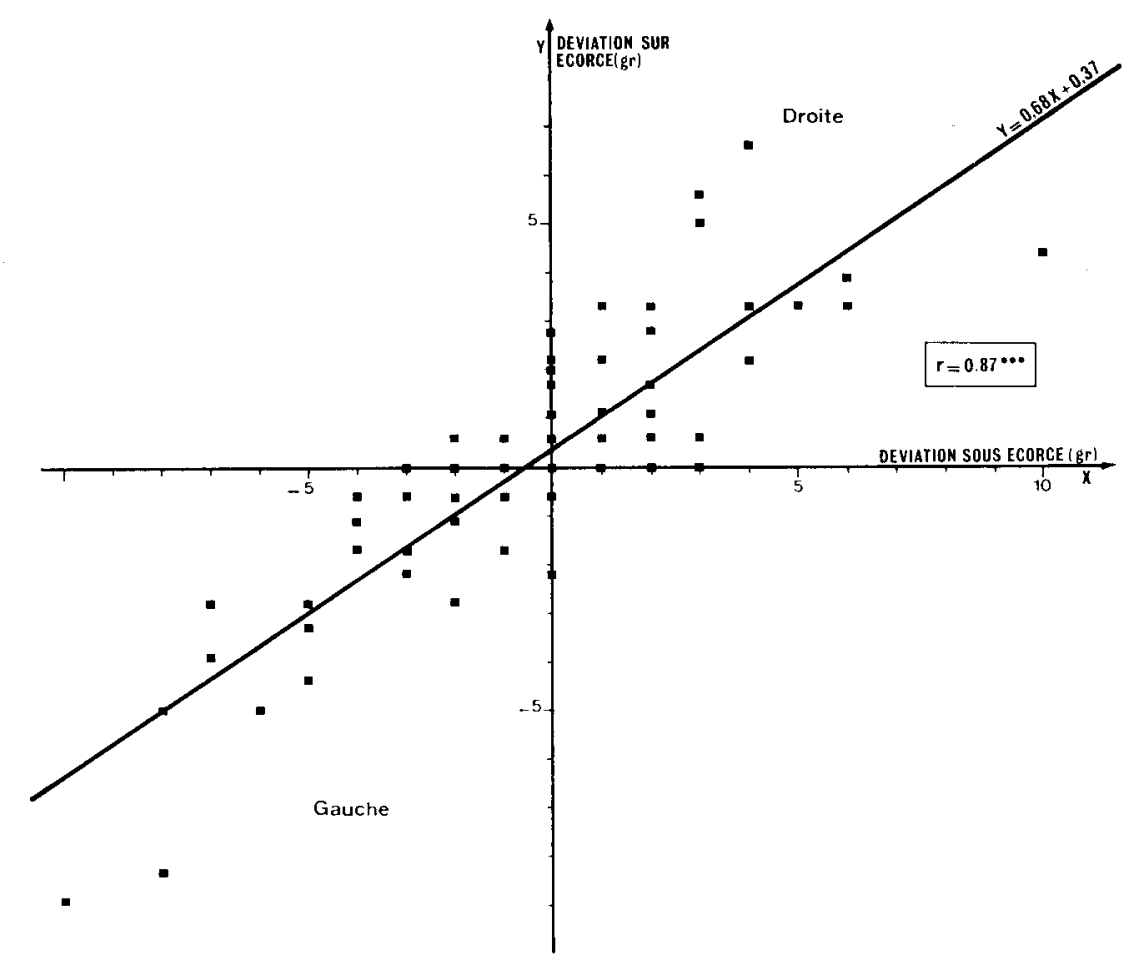

FIG. 7. - Chêne : coefficient de corrélation ef droite de régression entre valeurs d'angles sur écorce et sous écorce, tous niveaux confondus. Un certain nombre de points sont confondus.

Oak : correlation coefficient and regression line between outside

and inside bark fiber deviation values, including all measurement levels. Several points overlaps.

\section{3. - Eucalyptus dalrympleana}

3.31. Variabilité de l'angle du fil du bois.

Sur les cent seize arbres pris au hasard dans le test de descendance, 47,3 p. 100 (cf. fig. 8) ont un angle inférieur à 5 grades à $1,30 \mathrm{~m}$ du sol. Le nombre d'arbres ayant un angle supérieur ou égal à 10 grades est de 32 soit 28 p. 100, ce qui est élevé. En général, les déviations sont à droite $(75$ p. 100) ; seulement 9 p. 100 des arbres présentent une déviation à gauche. L'angle du fil du bois semble dans l'expérience peu influencé par les variations du milieu, l'effet bloc n'étant pas statistiquement différent de zéro. 


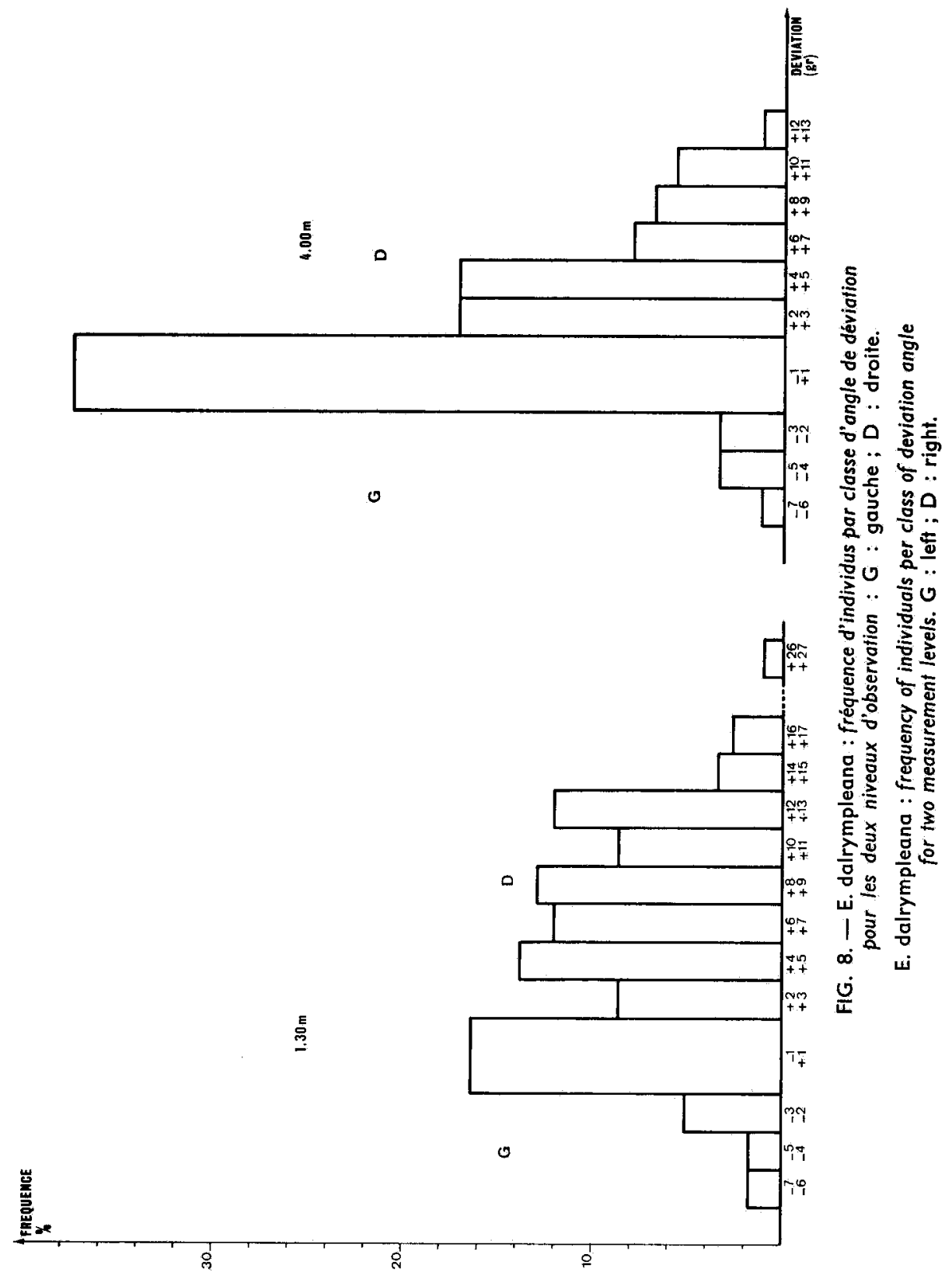


3.32. Validité de l'observation de l'écorce pour estimer la déviation de l'angle du fil du bois sous-jacent.

Trente six arbres avaient été sélectionnés dans différentes provenances pour leur résistance au froid, leur forte vigueur et leur faible angle du fil du bois. Pour ce dernier critère, l'examen de l'angle du fil du bois sur écorce semble avoir été efficace puisque 72 p. 100 des arbres présentent une déviation inférieure à 5 grades (contre 47,3 p. 100 dans le cas d'arbres pris au hasard dans une provenance donnée : cf. $\S$ 33.1). Par ailleurs, seulement 8 p. 100 des arbres présentent un angle supérieur ou égal à 10 grades. Le seul examen de l'écorce permet donc d'éliminer les arbres présentant de fortes déviations sinon en totalité du moins pour la plupart.

\subsection{Evolution de l'angle du fil du bois des cernes extérieurs avec la hauteur.}

La valeur moyenne de la déviation à 1,30 m est de 5,48 grades et à 4 m de $2,67 \mathrm{gra}$ des. Le test $t$ (méthode des couples) montre que ces deux valeurs sont significativement distinctes, au seuil de 1 p. 100. Le contraste d'angle du fil du bois, sur la partie observée du fût est donc en moyenne de 2,30 grades ce qui n'est pas considérable. Au niveau individuel cependant, on note une grande variabilité puisque les valeurs du contraste oscillent entre 0 ef 14 grades. Cette dernière valeur est importante sur une longueur de $3 \mathrm{~m}$ de bille. A $4 \mathrm{~m}$, on ne note plus qu'une fréquence de $7 \mathrm{p} .100$ d'individus présentant un angle du fil du bois supérieur à 10 grades.

Compte tenu de la nullité des effets blocs et de la structure du matériel végétal (famille, individus) on a effectué une analyse de variance-covariance à 1 facteur contrôlé (famille) pour les deux caractères angle du fil du bois à 1,30 m et $5 \mathrm{~m}$, selon le tableau 2.

\section{TABLEAU 2}

E. dalrympleana : analyse de variance $\sigma_{\mathrm{w}}^{2}$ ef $\sigma_{\mathrm{f}}^{2}$ sont respectivement les variances intra et inter et $k$ est le nombre "moyen " d'individus par famille

E. dalrympleana : variance analysis

$\sigma_{\mathrm{w}}^{2}$ and $\sigma_{\mathrm{f}}^{2}$ being respectively within and between progeny variance and $\mathrm{k}$ mean number of trees per progeny

Origine de la variation (Origin of variation) ddl (df) Carrés moyens (Mean squares)

$\begin{array}{lcc}\text { Famille (Progeny) } \ldots \ldots \ldots \ldots \ldots \ldots \ldots \ldots \ldots & 9 & \sigma_{\mathrm{w}}^{2}+k \sigma_{\mathrm{f}}^{2} \\ \text { Individu (Individual) } \ldots \ldots \ldots \ldots \ldots \ldots \ldots & \frac{78}{87} & \sigma_{\mathrm{w}}^{2}\end{array}$

Les calculs ont été faits grâce au programme UNFAL de la station de Biométrie. Les résultats montrent que la corrélation « intra » entre niveaux est globalement significative : $r=0,38 * *$. On peut donc " prédire » avec une faible précision cependant, la valeur de l'angle du fil du bois à $4 \mathrm{~m}$, à partir de la mesure effectuée à 1,30 $\mathrm{m}$. La 
corrélation « inter 》 (entre moyennes de familles) est $r=0,61$ NS. Cette valeur n'est toutefois pas très éloignée du seuil de signification à 5 p. 100. A l'intérieur de chaque famille, les corrélations sont généralement nulles (faible nombre de degrés de liberté), excepté pour une famille où $r=0,84 * *$.

\subsection{Hérédité de l'angle du fil du bois.}

L'analyse de variance à un facteur contrôlé (UNFAL) montre que l'effet « famille » est significatif $\left(F=2,17^{*}\right)$ pour l'angle mesuré à $1,30 \mathrm{~m}$. Les valeurs des moyennes de familles oscillent entre 2,1 et 9,8 grades. En revanche, il n'y a pas de différence entre familles pour l'angle mesuré à $4 \mathrm{~m}$. La même analyse réalisée sur la variable contraste d'angle (c'est-à-dire différence entre l'estimation à $1,30 \mathrm{~m}$ ef à $4 \mathrm{~m}$ ) aboutit également à la même conclusion : les familles ne sont pas statistiquement différentes. On trouvele même résultat négatif si l'on prend la moyenne des deux valeurs à 1,30 $\mathrm{m}$ et à $4 \mathrm{~m}$.

Enfin, on ne trouve pas de différence significative entre familles lorsque l'analyse de variance est effectuée sur l'angle du fil du bois mesuré à 1,30 m sur les cent seize individus (dont quatre-vingt-huit sont représentés dans l'étude portant sur deux niveaux de mesures).

En conclusion, l'hérédité du caractère angle du fil du bois apparaît faible à modérée. Toutefois, et compte tenu du mode de pollinisation partiellement entomophile des Eucalyptus, il est difficile de faire des hypothèses sur l'additivité ou la non-additivité du déterminisme génétique de ce caractère.

\section{4. - Discussions, perspectives}

\section{1. - Variabilité a 1,30 m.}

Pour le Hêtre, les valeurs extrêmes atteignent : 7 grades à gauche et 13 grades à droite. Ces valeurs sont rares ; ceci confirme l'opinion générale des forestiers pour qui la fibre torse est un défaut peu fréquent chez le Hêtre.

Pour le Chêne, les valeurs extrêmes de 10 grades à gauche ou à droite ne sont atteintes que rarement alors que la fibre torse est considérée comme un défaut fréquent chez cette espèce. II ne semble pas exclu que le peuplement choisi ne représente ni l'ampleur ni la variabilité du phénomène tel qu'il est connu. Ceci confirme d'ailleurs au passage la valeur du chêne de Bercé, mais nous incite à reprendre l'étude dans une population où le défaut est plus marqué.

Les valeurs extrêmes atteintes chez $E$. dalrympleana sont élevées : 7 grades à gauche et 27 grades à droite. Si le caractère héréditaire de ce défaut est confirmé, un effort important de sélection sera utile pour la production de variétés améliorées.

Remarquons une tendance pour une déviation en majorité à droite de la fibre torse chez $E$. dalrympleana et le hêtre, comme chez les résineux adultes. Le chêne ne présente pas de tendance nette.

\section{2. - Variabilité avec la hauteur}

Une hypothèse a été émise concernant le déferminisme de la fibre torse. Les substances hormonales produites par le houppier induiraient une inclinaison des 
fibres, et cela, d'autant plus que leur concentration est élevée (Maddern-Harris, 1973). Si l'on admet que dans les parties supérieures du fût cette concentration est plus élevée qu'en bas, on aurait dans un même cerne, une inclinaison des fibres croissant avec la hauteur. Dans notre échantillon, seuls les six hêtres déviant à gauche confirment cette hypothèse.

Pour la même raison, l'évolution dans le temps à un niveau donné (du cœur vers la périphérie) serait parallèle ; ce qui est confirmé par certaines observations réalisées sur le Douglas (Elliot, 1967). Des informations concernant cette dernière variation nous sont fournies aussi par Knigge ef Schulz (1959) pour le Chêne et le Hêtre. Les trois échantillons de Chêne analysés par ces chercheurs allemands montraient une variation anarchique de l'inclinaison de la fibre, passant de gauche à droite ou de droite à gauche plusieurs fois au cours de la vie de l'arbre. Chez le Hêtre, par contre, le phénomène semblait plus structuré. Au cœur de l'arbre l'inclinaison était en général faible. Elle augmentait en amplitude vers la droite ou vers la gauche. Dans nos échantillons, seuls des hêtres présentant de la fibre torse à droite vérifient cette hypothèse : diminution de l'inclinaison, vers le haut de l'arbre et vers son centre. Par ailleurs, au plan individuel, on observe des contrastes d'angle du fil du bois entre niveaux qui sont faibles pour le Chêne et le Hêtre mais beaucoup plus élevés pour E. dalrympleana.

Enfin, à la différence des conifères, les corrélations entre niveaux d'observation sont élevées surtout chez le Chêne et le Hêtre.

\section{3. - Validité de la mesure sur écorce}

Les échantillons des trois espèces étudiées montrent la bonne concordance tout au moins sur les grands nombres - de l'observation sur écorce avec celle des cernes extérieurs du bois. Cette constatation, qui semble couler de source, mérite quelques précisions. Les couches annuelles successives se forment dans le bois et dans l'écorce, en sens inverse à partir de l'assise libéro-ligneuse ; dans les arbres à écorce persistante, tels que le Chêne et le Hêtre, la surface de l'écorce correspond donc au bois le plus anciennement formé (bois situé à proximité de la moelle), et non aux couches les plus superficielles de celui-ci ; ainsi, les bonnes corrélations trouvées ne correspondent nullement à une évidence et laissent à penser que les variations de l'angle de déviation avec l'âge sont finalement de faible amplitude. Knigge et Schulz (op. cit.), tout en étant d'accord avec cette vue des choses, ne trouvent pas cette concordance. Ils ont travaillé sur trois arbres par espèce, effectif faible pour en tirer des lois, la variation de la déviation de la fibre étant très grande en fonction de l'âge.

\section{4. - Part de l'hérédité dans le déterminisme de la fibre torse}

Chez le Hêtre et le Chêne, les informations que nous possédons nous viennent essentiellement de Krahl-Urban (1953 ef 1961). Ce forestier allemand a d'abord remarqué que les arbres présentant de la fibre torse ont fréquemment des branches qui modifient leur orientement d'une façon homogène dans la cime; elles s'enroulent dans un même sens autour de l'axe du tronc. Il utilise cette observation comme test pour déterminer l'aptitude à la fibre torse chez de jeunes plants. D'après cet auteur, les descendances d'arbres à fibre torse présentent en moyenne deux fois plus de 
branches enroulées que celles d'autres arbres. Par ailleurs, il a noté dans certains peuplements des groupes d'arbres à fibre torse dans un même sens de rotation. II fait l'hypothèse que ces arbres sont issus d'un même semencier. Mais, en forestier avisé, il n'exclut pas l'effet du milieu ; plus tard, au moyen d'une plantation comparative de descendances maternelles, il élimine l'hypothèse de cet effet.

Les résultats présentés ne nous apportent des informations que chez $E$. dalrympleana. Cette espèce, ou tout au moins l'échantillon analysé, ne révèle qu'un faible effet famille et cela uniquement à $1,30 \mathrm{~m}$. Cette faiblesse vient principalement d'une variabilité intradescendances qui est très élevée.

Désireux d'approfondir cette question de l'hérédité de ce caractère, notammen† chez le Hêtre, nous avons proposé à nos collègues allemands de Basse-Saxe, gestionnaires de ces tests de descendances, d'y effectuer des mesures par la méthode des radioisotopes. Nos propres études de structure génétique sur le Chêne et le Hêtre fourniront à l'avenir un matériel de choix pour l'étude de l'hérédité de ce caractère.

\section{5. - Conclusion}

Cette étude nous a permis de constater que chez les trois espèces, l'angle du fil du bois est variable, que l'aspect de l'écorce rend compte de la déviation sous-jacente du bois et que, tout au moins chez le Chêne et le Hêtre, une observation à n'importe quel niveau rend bien compte de celle de l'ensemble du fût. Des possibilités de sélection massale sont ouvertes, sous réserve d'héritabilités génotypique et génétique suffisamment élevées. Cette sélection pourra être réalisée à l'œil nu avec, pour certains hêtres, la nécessité d'une observation assez approfondie.

L'appréciation de l'effet famille sur l'angle du fil du bois chez E. dalrympleana ne montre qu'une tendance qui mériterait vraisemblablement d'être clarifiée dans quelques années.

Deux questions restent à approfondir à notre avis :

- évolution de l'angle du fil du bois à un niveau donné du cœur vers la périphérie de l'arbre,

- héritabilité du caractère aussi bien au niveau génotypique, par exemple pour le programme de multiplication végétative du Chêne, qu'au niveau génétique (transmission parents-enfants cas de la régénération naturelle par exemple).

Si on veut obtenir une réponse claire et précise à la première question, l'étude doit être réalisée sur un grand nombre d'individus, elle doit être de ce fait non destructive. Les études préliminaires réalisées sur carottes de conifères par la Station de Recherches sur la Qualité du Bois du C.N.R.F. à Nancy, se sont heurtées jusqu'à maintenant à des difficultés insurmontées. Si elles n'aboutissent pas, il devra être envisagé dans certains tests de clones ou de descendances, de réaliser des mesures périodiques d'angle de déviation de la fibre pour suivre cette évolution.

La réponse à la deuxième question est subordonnée à la première, mais elle nécessite en plus l'estimation des héritabilités à plusieurs âges pour déterminer celui auquel la sélection serait la plus efficace. 


\section{Remerciements}

Les informations fournies par $M$. Venet, la lecture critique de MM. Polge et Lafouge ont contribué à la clarification d'un certain nombre de points et à la mise en forme de ce rapport.

Les mesures sur le terrain ont été exécutées avec la collaboration de MM. Piermant ef Aliberti. Que tous soient ici vivement remerciés.

\section{Summary}

Variability of fiber deviation angle in three hardwood species : Fagus silvatica, Quercus petraea and Eucalyptus dalrympleana

Spiral grain, in most wood utilizations is considered as a severe defect. Variability of that characteristic has been studied in two broadleaf species, Fogus silvatica and Quercus pefraea which are of great importance in French forests and in Eucalyptus dolrympleana which has a good potential for reforestation in southeastern France. Two methods were used to assess fiver deviation. Both are non destructive. The first consists in injecting into the outer wood rings a radioisotope ${ }^{42} \mathrm{~K}$, during a period of intense sap rising, and trace its progression with autoradiographic films applied on the bark. The second, only applicable to Beech and Oak was to mesure angles between direction of bark fissures, slots or hollows on the one hand and bole generating lines on the other hand. The main results and consequences are (1). Fiber deviation at breast height may be important for individual E. dalrympleana. Attention will have to be given to this in breeding programs. High deviations seldom occur in Beech and Oak. The sampled Oak frees were probably of higher quality for that characteristic than is common in the range of the species (2). Fiber deviation varies from one level to another within trees. This variation is high for $E$. dalrympleana and fairly low for Beech and Oak (3). Correlations between observations at different levels are significant. Fiber deviation at any level on the bole will enable a good estimation at all levels (4). Bark deviation is largely connected to outer rings fiber deviation. External observations may thus be considered reliable (5). A significant but low genetic effect has been found for $E$. dalrympleana. More information is needed for inheritance of fiber deviation and variation according to tree age.

\section{Références bibliographiques}

ARBEZ M., BARADAT Ph., BIROT Y., 1978. Variabilité et hérédité de l'angle du fil du bois mesuré à l'aide d'un traceur radioactif chez le Pin maritime (Pinus pinaster Ait.) et le Pin laricio (Pinus nigra Arn. Jaricio). J. Can. Rech. Forest., 8 (3), 280-289.

BIROT Y., ARBEZ M., AZOEUF P., et HOSLIN R., 1979. Variabilité phénotypique de l'angle du fil du bois en fonction de la hauteur chez le Pin laricio et le Douglas. Ann. Sci. forest., 36 (2), 9 p.

ELLIOT G. K., 1967. Some problems of spiral grain with special references to conifers. $14^{\mathrm{e}}$ Congrès IUFRO Munich 1967. Groupe de Travail 22/41, 413-435.

FERRANDES P., 1970 . Les Eucalyptus en zone méditerranéenne française. Choix des espèces. Premiers résultats d'essais de provenances. Rev. Forest. Franç., no 3, 371-377.

KELLER R., AZOEUF P., ef HOSLIN R., 1974. Détermination de l'angle de la fibre torse d'arbres sur pied à l'aide d'un traceur radioactif. Ann. Sci. forest., 31 (3), 161-169.

KNIGGE W. ef SCHULZ H., 1959. Methodische untersuchungen über die Möglichkeit der Drehwuchsfeststellung in verschiedenen Alterzonen von Laubhölzern. Holz als Roh und Werksioft. 17 (9), 341-351.

KRAHL-URBAN J., 1953 ; Drehwuchs bei Eichen und Buchen. Allgemeine Forszeirschrift 40, 540549.

KRAHL-URBAN J., 1961. Über den Drehwuchs bei Buchen. Forstarchiv, 32 (10), 197-201.

MADDERN-HARRIS J., 1973. Spiral grain and xylem polarity in Radiata pine : microscopy of cambial reorientation, IUFRO Meefing Sect 41, Capeteown, 15 p.

VENET J., 1970. Cours de l'Ecole Nationale du Génie Rural des Eaux et des Forêts. Nancy, 231 p. 


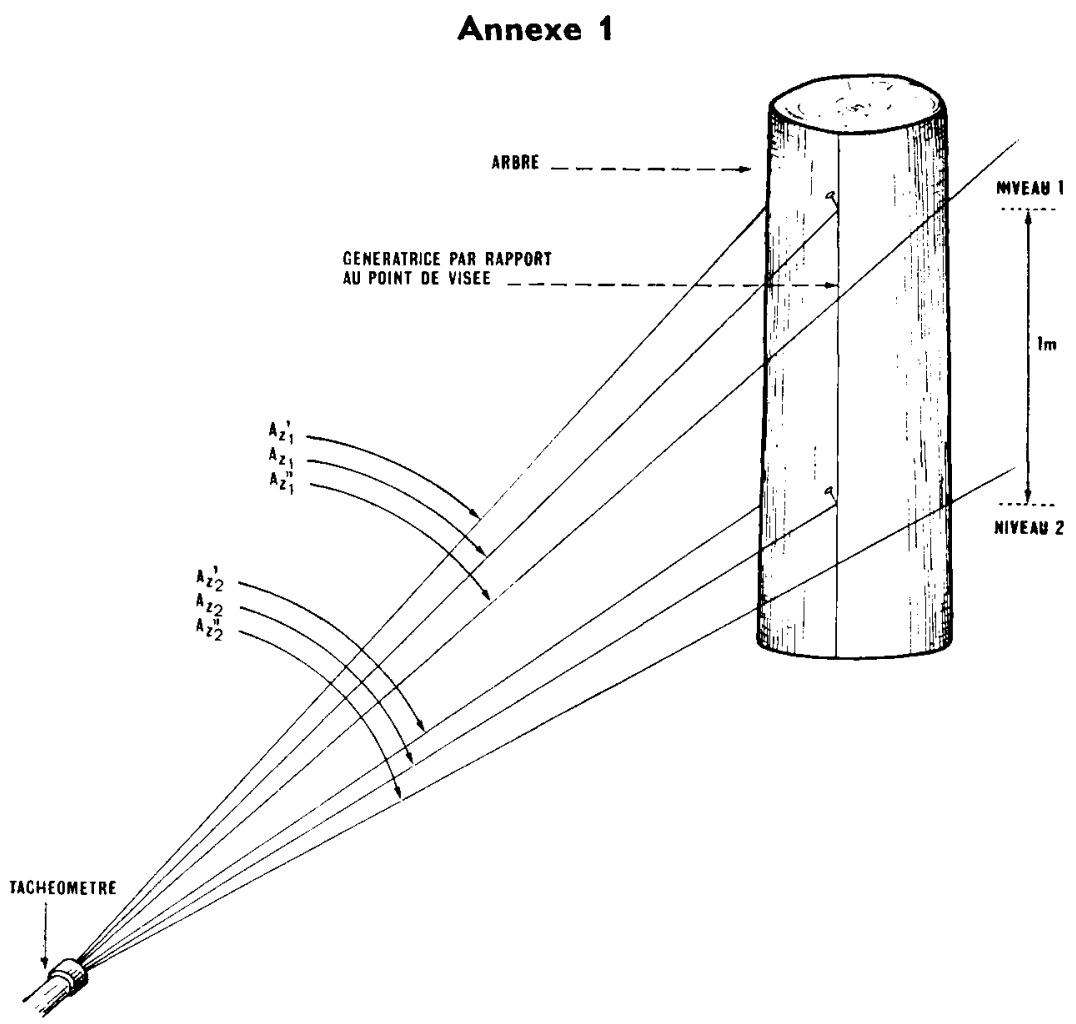

Au niveau « 1 », les azimuts des tangentes au tronc par rapport au point de visée sont déterminés. Celui de la génératrice est la moyenne des deux :

$$
A z_{1}=\frac{A z_{1}^{1}+A z_{11}^{1}}{2}
$$

L'axe de visée est alors fixé à la valeur $\mathbf{Z} z_{1}$, un clou est planté au point correspondant du tronc. Il est procédé de même pour le niveau « 2 ». Une règle, appuyée sur les deux clous, permet le tracé de la génératrice.

At level 1 bearings of tangents to bole from sight point are taken $A z_{1}^{\prime}$ and $A z_{1}^{\prime \prime}$. Bearing of generating line is the arithmetic mean :

$$
A z_{1}=\frac{A z_{1}^{\prime}+A z_{1}^{\prime \prime}}{2}
$$

The sight line is fixed on the value $A z_{1}$. A nail is planted on the corresponding point of the bole. The same process is done at level 2 .

A rular applied on the nails enables drawing the generating line. 